\title{
Hospital volume and patient outcomes after cholecystectomy in Scotland: retrospective, national population based study
}

\author{
(c) $(1)(9)$ OPEN ACCESS
}

\begin{abstract}
Ewen M Harrison lecturer in surgery ${ }^{1}$, Stephen O'Neill research fellow ${ }^{1}$, Thomas S Meurs medical student $^{2}$, Pang L Wong medical student ${ }^{1}$, Mark Duxbury clinician scientist and honorary consultant surgeon ${ }^{1}$, Simon Paterson-Brown consultant surgeon and honorary senior lecturer ${ }^{1}$, Stephen J Wigmore professor of transplantation surgery ${ }^{1}$, O James Garden regius professor of clinical surgery ${ }^{1}$
\end{abstract}

${ }^{1}$ Department of Clinical Surgery, University of Edinburgh, Royal Infirmary of Edinburgh, Edinburgh EH16 4SA, UK; ${ }^{2}$ Department of Surgery, Leiden University Medical Centre, Leiden, Netherlands

\begin{abstract}
Objectives To define associations between hospital volume and outcomes following cholecystectomy, after adjustment for case mix using a national database.

Design Retrospective, national population based study using multilevel modelling and simulation.

Setting Locally validated administrative dataset covering all NHS hospitals in Scotland.

Participants All patients undergoing cholecystectomy between 1 January 1998 and 31 December 2007.

Main outcome measures Mortality, 30 day reoperation rate, 30 day readmission rate, and length of stay.

Results We identified 59918 patients who had a cholecystectomy in one of 37 hospitals: five hospitals had high volumes (>244 cholecystectomies/year), 10 had medium volumes (173-244), and 22 had low volumes $(<173)$. Compared with low and medium volume hospitals, high volume hospitals performed more procedures non-electively (17.1\% and $19.5 \%$ v $32.8 \%$ ), completed more procedures laparoscopically (64.7\% and $73.8 \% v 80.9 \%)$, and used more operative cholangiography $\left(11.2 \%\right.$ and $6.3 \% \vee 21.2 \% ; \chi^{2}$ test, all $\left.\mathrm{P}<0.001\right)$. In a well performing multivariable analysis with bias correction for a low event rate, the odds ratio for death was greater in both the low volume (odds ratio $1.45,95 \%$ confidence interval 1.06 to $2.00, \mathrm{P}=0.022$ ) and medium volume $(1.52,1.11$ to $2.08, P=0.010)$ groups than in the high volume group. However, in simulation studies, absolute risk differences between volume groups were clinically negligible for patients with average risk (number needed to treat to harm, low $v$ high volume, 3871, 1963 to 17 118), but were significant in patients with higher risk. In models
\end{abstract}

accounting for the hierarchical structure of patients in hospitals, those in medium volume hospitals were more likely to undergo reoperation (odds ratio $1.74,1.31$ to $2.30, \mathrm{P}<0.001$ ) or be readmitted $(1.17,1.04$ to $1.31, P=0.008$ ) after cholecystectomy than those in high volume hospitals. Length of stay was shorter in high volume hospitals than in low (hazard ratio for discharge $0.78,0.76$ to $0.79, \mathrm{P}<0.001$ ) or medium volume hospitals $(0.75,0.74$ to $0.77, \mathrm{P}<0.001)$. These differences were also only of clinical significance in patients at higher risk.

Conclusions There is wide variation among hospitals in the management of gallstone disease and an association between higher hospital volume and better outcome after a cholecystectomy. The relative risk of death is lower in high volume centres, and although absolute risk differences between volume groups are significant for elderly patients and patients with comorbidity, they are clinically negligible for those at average risk.

\section{Introduction}

The variation in outcome after surgery and its association with the volume of patients treated by an institution or individual surgeon has been extensively examined over the past 50 years. These studies usually focus on specialist interventions (such as cancer resections or cardiovascular procedures) and the results are often used to argue for the centralisation of surgical services ${ }^{1}$ and an increase in the subspecialisation of surgeons. ${ }^{2}$ Individual surgeon volume is important ${ }^{3}$ but is a less consistent predictor of outcome than hospital volume. ${ }^{4}$ Although volume can predict outcome, it is not necessarily a good surrogate for quality. Possible explanations for the volume effect include differences in institutional structure, such as staffing levels, ${ }^{5}$ and variation 
in processes, such as the "failure to rescue" patients from complications. ${ }^{6}$

Within this debate, less attention has been given to high volume, general surgical procedures with a low risk, possibly because robust outcomes in administrative databases are harder to identify. In addition, since measureable outcomes (such as mortality) are better after surgery of this type, effect sizes tend to be small and could be regarded as less clinically relevant. Yet, in view of the large number of cholecystectomies performed in developed countries each year, even relatively rare adverse events contribute considerably to morbidity. Indeed, a study examining the contribution of different surgical procedures to total inpatient morbidity ranked inpatient cholecystectomy third (6\%) after colectomy and small bowel resection. ${ }^{7}$

Despite this, the relation between hospital volume and cholecystectomy remains unclear. In a historical study of open cholecystectomy, no link was shown between hospital volume and mortality. ${ }^{8}$ Similarly, in a large contemporary series of patients undergoing laparoscopic cholecystectomy in the United States, researchers found no association between hospital volume and the risk of major complication or death, although open conversion was associated with low volume centres. ${ }^{9}$ In the acute setting where complications are more common and outcome differences can be more prominent, higher volume surgeons were associated with shorter lengths of stay and fewer open conversions, ${ }^{10}$ but again no association with hospital volume was shown.

Using a high quality national dataset which encompasses all emergency and elective surgical procedures, we aimed to evaluate four independent measures of patient outcome after cholecystectomy and determine their association with hospital volume. We characterised this link further with risk modelling to determine the effect of age, comorbidity, and socioeconomic status.

\section{Methods}

\section{Study design, setting, and participants}

We did a retrospective, population based study using data from the Information Services Division of NHS Scotland. We identified all NHS patients undergoing cholecystectomy between 1 January 1998 and 31 December 2007 in Scotland. Episode records including all previous and subsequent encounters were retrieved up to 31 December 2008 (web appendix, page 1).

We excluded patients from further analysis if the diagnosis relating to the index procedure was related to carcinoma or trauma (web appendix, page 2), or if the index procedure was carried out in a paediatric or private hospital (web appendix, page 3). We accounted for hospitals amalgamating or changing name during the study period.

The study was performed in accordance with the Strengthening the reporting of observational studies in epidemiology (STROBE) guidelines. ${ }^{11}$ The National Services Scotland Privacy Advisory Committee approved the study. All patient data were anonymised. The primary investigator is a registered data controller with the United Kingdom Information

Commissioner's Office and all data were treated in accordance with the principles of the Data Protection Act 1998.

\section{Data validation and bias}

The Information Services Division database was internally consistent and we did no data imputation (missing data related only to deprivation scoring). We matched a three year sample with a centrally administered, operating theatre database, and the concordance of matched cases was $89 \%$. The coding of laparoscopic to open conversions was inconsistent in earlier years, and consequently we classified all these procedures as open. The identities of the operating and responsible surgeons were poorly correlated ( $50 \%$ concordance) and were not suitable for surgeon volume analysis. In a second analysis, we matched cases to a locally held audit database and saw a good correlation for reoperation rate $(98 \%)$, readmission rate $(97 \%)$, and length of stay $(94 \%)$.

\section{Factors and covariates}

We defined hospital volume as the mean number of cholecystectomies performed per hospital per year in 1998-2007. In a manner similar to Birkmeyer and colleagues, we evaluated hospital volume as a continuous (log transformed) variable in the assessment of statistical significance. We then created categorical variables by ranking institutions in order of increasing volume and selecting cut-off points that most closely sorted patients into three evenly sized groups with low, medium, and high volume (web appendix 3 ). ${ }^{3}$ No hospitals were excluded. We did sensitivity analyses using different cut-off points and an alternative 10 year cohort (1997-2006), from which we saw no changes in the significance of model parameter estimates for differences between hospital volume groups.

We explored models of morbidity scoring using disease codes from ICD-9 and ICD-10 (international classification of diseases, 9th and 10th revisions) from all previous healthcare encounters, including the Deyo modification of the Charlson score and the Elixhauser score. ${ }^{12}$ No single method resulted in better model performance, and Charlson score was used in the final analysis (web appendix, page 3). ${ }^{3}$ The Scottish Index of Multiple Deprivation (SIMD) 2009 uses an improved methodology to provide a relative measure of deprivation in Scotland. Patients' postcodes at index procedure determined SIMD quintiles, which were considered as a continuous variable. An SIMD score could not be assigned for 271 patients, because they were not included in models using SIMD. We found no significant patterns between these 271 patients and the remainder of the cohort for other variables or outcome measures.

\section{Outcome measures}

We determined patient death by using probability matching, record linkage procedures between patient episodes from the Information Services Division and by using death records from the Registrar General of Scotland. Mortality was defined as death occurring within 30 days of the index procedure or before discharge (censored at 120 days). We defined 30 day reoperation as the occurrence of any procedure in the 30 days after index cholecystectomy involving the upper digestive tract (Office of Population Censuses and Surveys version 4, code G) or other abdominal organs (principally digestive; code J). We defined 30 day readmission as an emergency readmission to any Scottish hospital within 30 days of the date of the index procedure. Length of stay was defined as the period from the date of index procedure to the discharge date of continuous inpatient stay (that is, a patient who was directly transferred to another hospital was not classified as being discharged).

\section{Statistical procedures}

We examined initial univariable associations using $\chi^{2}$ tests and one way analysis of variance for categorical and continuous predictors, respectively. All $\mathrm{P}$ values were two tailed. We initially specified binary logistic regression models conditionally, using backwards likelihood ratio methods. 
However, in all included models, factors and covariates found to be significant in univariable models were also significant in multivariable models. We determined goodness of fit and included the Hosmer-Lemeshow test and predictive performance quantified by the area under the receiver operator characteristic curve (c statistic). The ratio of predictors to events was never lower than 15:1.

We used bootstrap methods to derive confidence intervals in multivariable models unless otherwise indicated. We specified hierarchical logistic regression models to account for the clustering of patients within hospitals. In all cases, random coefficient models were not significantly better than random intercept models (as determined by likelihood ratio tests). For mortality outcomes, the multilevel model was no improvement on the fixed effects model; however, since death after cholecystectomy was regarded as a rare event, we used a model including a bias correction. ${ }^{13}$ Length of stay in cholecystectomy was particularly right skewed, making analysis difficult. The problems of ordinary least squares and regression methods, even using a log transformed dependent variable, are well described. ${ }^{14}$ We used a Cox proportional hazards procedure to model the risk of discharge. The underlying hazard function was assessed and seen to be constant, and no time dependent variables were specified. Page 8 of the web appendix shows a generalised linear model for comparison.

Finally, to provide a real world interpretation of the data, we simulated predicted probabilities and absolute risk differences for different risk strata using the fixed effect models (asymptotic normal approximation to the log likelihood, 1000000 simulations per quantity of interest; web appendix, page 4). ${ }^{15}$ In particular, these procedures rely on the correct specification of interactions between variables. We assessed all two way interactions and identified no significant interactions in the final models.

We specified the logistic regression models in Stata SE 11.0 (StataCorp) using commands logit and xtmelogit. We did rare event logistic regression $\left(\right.$ relogit $\left.^{16}\right)$, generalised linear modelling, Cox proportional hazards modelling, and risk modelling in $\mathrm{R}$ 2.11.1 (R Foundation for Statistical Computing) using the Zelig ${ }^{17}$ and Survival packages.

\section{Results}

We identified 60732 individuals as having undergone a cholecystectomy between 1 January 1998 and 31 December 2007. Patients were excluded if the primary diagnosis was cancer (263) or trauma (12) or if the cholecystectomy was performed in one of eight private hospitals (457) or one of four paediatric hospitals (82), giving a final dataset of 59918 patients and 37 hospitals. Patients were equally divided across three bands of hospital volume: high volume ( $>244$ procedures/year; five hospitals, 18425 patients), medium volume (173-244; 10, 20 $534)$, and low volume $(<173,22,20959)$.

\section{Patient and hospital characteristics}

The annual total number of cholecystectomies increased from 5131 in 1998 to 7259 in 2007 (fig $1 \Downarrow$ ). The proportional increase over this time was greater for non-elective procedures $(2.25$ times) than for elective procedures (1.25 times). This increase was confined to the group of high volume hospitals, with static annual rates in low and medium volume hospitals (figs $2 \Downarrow$ and $3 \Downarrow$ ). Until 2002, most cholecystectomies were performed in low volume centres, but after 2005 , the number in the high volume group exceeded that of the other two for the first time (web appendix, page 7). The proportion of non-elective cholecystectomies increased from $16.7 \%$ in 1998 to $26.6 \%$ in 2007. However, this proportion varied greatly between hospitals, ranging from $0.26 \%$ to $53.3 \%$. Taking the period as a whole, the proportion of non-elective cholecystectomies was $32.8 \%$, $19.5 \%$, and $17.1 \%$ in high, medium, and low volume hospitals, respectively ( $\chi^{2}$ test, $\mathrm{P}<0.001$; table $1 \Downarrow$ ). Levels of deprivation were greater (SIMD score 1 and 2) in medium volume hospitals $(54.8 \%)$ than in low volume $(42.9 \%)$ or high volume hospitals $(39.4 \%)$. Levels of comorbidity were low in this patient population with little difference between groups.

The likelihood of the procedure being completed laparoscopically rose with increased hospital volume $(\mathrm{P}<0.001)$, as did the use of operative cholangiogram $\left(\chi^{2}\right.$ test, $\mathrm{P}<0.001$; table $2 \Downarrow$; web appendix, page 9 shows results from multivariable models). Although differences were significant between volume groups in rates of endoscopic retrograde cholangiopancreatography and bile duct exploration, the differences in magnitude were small.

\section{Outcomes}

\section{Mortality}

Unadjusted overall 30 day and inpatient mortality after cholecystectomy was $0.49 \%$. On univariable analysis, mortality in the medium volume group $(0.55 \%, \mathrm{P}=0.033)$ but not in the low volume group $(0.51 \%, \mathrm{P}=0.094)$ was greater than that in the high volume group $(0.40 \%$; tables $3 \Downarrow$ and $4 \Downarrow)$. Mortality was higher for non-elective procedures than for elective procedures in the low, medium, and high volume groups (79/3585 (2.20\%), 81/4010 (2.02\%), and 56/6044 (0.93\%) $v$ 28/17 $374(0.16 \%), 31 / 16524(0.19 \%)$, and 17/12 $381(0.14 \%)$, respectively). All variables that were significant in the univariable model maintained significance in a multivariable logistic regression model, which fitted the data well (c statistic 0.92). When considered as a log transformed continuous variable, hospital volume was significantly related to mortality $(\mathrm{P}=0.0267)$. As a categorical variable, volume was significant in both the low volume group (odds ratio $1.45,95 \%$ confidence interval 1.06 to $2.00, \mathrm{P}=0.022$ ) and medium volume group (1.52, 1.11 to $2.08, \mathrm{P}=0.010$ ), compared with the high volume group (table 4). A hierarchical multilevel analysis did not improve the model and we saw no alteration in parameter estimates or their standard errors. In view of the low ratio of events (deaths) to hospitals, it is unsurprising that we found almost no variance attributed to the hospital level in the model (likelihood ratio test, multilevel $v$ fixed effect, $\mathrm{P}=1.0$ ).

We used simulation procedures to provide reliable estimates of expected probabilities of adverse outcomes in different patient groups (table $5 \Downarrow$, fig $4 \Downarrow$ ). The left panel in figure 4 presents probabilities of adverse outcome for the most common type of patient undergoing cholecystectomy. For this low risk, standard group of patients undergoing elective cholecystectomy (table 5 , example 1), although relative risk differences were significant between the low and medium volume groups and the high volume group, the absolute risk differences in mortality were so small as to be clinically meaningless (low $v$ high volume comparison, $0.00026,95 \%$ confidence interval 0.00006 to $0.00051, \mathrm{P}=0.010$; equivalent to number needed to treat to harm of 3871,1963 to 17118 ). Adverse outcomes were more common in non-elective cholecystectomies than in elective procedures. When we modelled the low risk standard group (which also represents the most common type of patient undergoing non-elective cholecystectomy; table 5, example 2) in the non-elective setting, the absolute risk differences in mortality 
between low and high volume groups became more pronounced (0.0030, 0.0007 to $0.0059, \mathrm{P}=0.010 ; 338,171$ to 1491$)$.

As baseline risk increased, absolute risk differences became highly clinically significant (fig 2 , right panel). Table 5 provides examples of patients with different risk profiles. For instance, in example 7, a man older than 70 years with significant comorbidity presenting as an emergency and undergoing cholecystectomy has a $15-20 \%$ probability of death. At this level of risk, differences between hospital volume bands were pronounced, as shown by the numbers needed to treat to harm (low $v$ high volume comparison $17,95 \%$ confidence interval nine to 74; medium $v$ high volume 19, 10 to 143 ; table 5).

\section{Reoperation and readmission rates at 30 days}

The association between rates of reoperation and readmission and hospital volume was non-linear. The medium volume group had a greater number of reoperations than the low and high volume groups $(4.65 \%$ v $3.23 \%$ and $3.29 \%$, respectively; $\mathrm{P}<0.001)$, and a higher readmission rate than the low and high volume groups $(8.16 \% \vee 7.55 \%$ and $7.61 \%$, respectively, $\mathrm{P}=0.024$; table 3 ). These differences were more pronounced in a fixed effect multivariable model (web appendix, page 8). In the multilevel model, the medium volume group (odds ratio $1.74,95 \%$ confidence interval 1.31 to $2.30, \mathrm{P}<0.001$ ) but not the low volume group $(1.24,0.95$ to $1.62, \mathrm{P}=0.114)$ had a significantly higher reoperation rate than the high volume group (table 4). The model for readmission rate was less robust, but again we saw significant differences between the medium volume $(1.17,1.04$ to $1.31, \mathrm{P}=0.008)$ and high volume groups, but not between the low volume (1.09, 0.98 to $1.22, \mathrm{P}=0.110)$ and high volume groups.

\section{Length of stay}

Mean length of stay was shorter in the high volume group (mean 2.59 days) than in both the low volume (2.99) and medium volume (3.09) groups (both $\mathrm{P}<0.001$ ). Modelling length of stay was difficult, particularly since these data were extremely right skewed. Regressing simple log transformed length of stay against predictors resulted in a poor fit (that is, the model inadequately described the data). We successfully modelled length of stay using a Cox proportional hazards model, as has been suggested by others. ${ }^{14}$ Hospital volume as a continuous variable was highly significant $(\mathrm{P}<0.001)$. Patients were less likely to be discharged in the low volume (hazard ratio 0.78 , 0.76 to $0.79, \mathrm{P}<0.001)$ and medium volume $(0.75,0.74$ to 0.77 , $\mathrm{P}<0.001)$ groups than those in the high volume group (table 4$)$. Thus, at any given time, low and medium volume hospitals were discharging $22 \%$ and $25 \%$ fewer patients than high volume hospitals, respectively.

\section{Discussion}

This is the first study to our knowledge to show a significant association between hospital volume and multiple outcome measures after a low risk, high volume, general surgical procedure. Over a 10 year period, we saw an ongoing rise in the number of cholecystectomies performed in Scotland each year, a trend that was confined to high volume hospitals. In these centres, a higher proportion of cholecystectomies were performed in the non-elective setting, more were completed laparoscopically, and operative cholangiography was used more frequently. However, despite high volume hospitals having better outcomes than lower volume hospital, these differences might be viewed as negligible in practical terms.

\section{Strengths and weaknesses of the study}

This study used a high quality, locally validated dataset that controlled for the case mix in the assessment of outcomes after cholecystectomy. Scotland has a relatively stable population that, together with the national coverage of these data, allows for accurate outcome assessments such as the readmission of a patient to a hospital other than that of the primary procedure is accounted for. The statistical analyses were performed at length and with care, and the provision of simulated "real world" quantities of interest allowed us to draw clinically relevant conclusions. Although case mix was controlled with several factors, a weakness of studies using administrative databases is the inability to control for illness severity, particularly in acutely unwell patients. This persistent problem could explain some of the differences seen. In addition, although the national dataset performed well in the validation exercise, this was only carried out in one region of Scotland; unidentified geographical differences in dataset accuracy could exist. In this study, we have not included explanatory variables in hospital structure and process, which is the focus of ongoing work.

High volume hospitals had lower mortality rates and shorter lengths of stay after cholecystectomy than centres with low and medium volumes. However, reoperation and readmission rates were significantly higher in medium volume centres only. Although the observation of poorer outcomes in medium volume centres is consistent with the published literature, it is more difficult to explain why low volume centres had similar outcomes to high volume centres for these measures. This phenomenon has been described before, ${ }^{5}$ and it could have been artefactual in the present study, since reoperation and readmission models were less robust than the models for mortality and length of stay. If the effect is genuine, it could be that low volume centres are referring complex cases to tertiary centres or have adapted their structures and processes to compensate for a lower volume.

\section{Strengths and weaknesses in relation to other studies}

Unlike other studies, this study has clearly shown differences in outcomes related to hospital volume after cholecystectomy. It is an analysis of gallbladder operations undertaken in all public hospitals in Scotland over a 10 year period, whereas other studies could have been influenced by the institutions selected for analysis. The largest series identified looked specifically at complication rates after laparoscopic cholecystectomy in over one million US patients from the Nationwide Inpatient Sample (1998-2006). ${ }^{9}$ Although hospital volume was a significant predictor of complication in univariable analysis, it was not maintained in a multivariable analysis. However, the risk of open conversion remained significant (low $v$ high volume, odds ratio $1.32,95 \%$ confidence interval 1.18 to 1.49 ). This cohort included open conversions but did not include primary open procedures, which might have blunted any expected effect sizes. Yet this explanation cannot account for the differences shown in the present study, in which volume effects for all outcomes persisted in models including only procedures completed laparoscopically (web appendix, page 9).

A difficulty in comparing studies of hospital volume is the variability in the definition of low and high volume. Two such studies have examined the effect of volume on outcome, in which the annual procedure volume of included institutions was significantly lower than in the present study. Using the Veterans' Affairs National Surgical Quality Improvement Program database, Khuri and colleagues analysed eight major surgical 
procedures including open $(\mathrm{n}=7113)$ and laparoscopic $(\mathrm{n}=8602)$ cholecystectomy, and found no significant association between hospital procedure volume and 30 day mortality rate. ${ }^{18}$ Khuri and colleagues' selected cohort differed from our cohort by being older and predominately male, with the major difference in ranges of annual hospital volume: open cholecystectomy 1-39, laparoscopic cholecystectomy 0-44. All these centres would have been classified as low volume in the current study, making meaningful comparisons difficult.

Similarly, a smaller uncontrolled Norwegian study $(n=5343)$ found a linear association between hospital volume and a severe complications index, but again, mean hospital volume was significantly lower $(>50,25-50$, and $<25$ procedures/year for high, medium, and low volumes, respectively). ${ }^{19} \mathrm{~A}$ "threshold effect" could exist - that is, a volume level below which volume differences between hospitals have no measureable effect on outcome. This effect has been described for many specialist procedures, but is difficult to identify if event rates are low. We could not identify a threshold effect for any of the outcome measures in the current study.

Several studies have assessed the influence of surgeon volume and outcome after cholecystectomy. In 2000, McMahon and colleagues used earlier data from the same source as the current study and showed that surgeons with lower volumes were associated with reduced odds of a laparoscopic procedure, patients with a significantly longer postoperative stay, and for those performing fewer than three cholecystectomy a year, a substantial increase in the odds of postoperative death. ${ }^{20}$ As noted earlier, the surgeon volume metrics in this dataset was found to be unreliable for the period analysed in the current study. In another study from the Nationwide Inpatient Sample database examining patients undergoing cholecystectomy for cholecystitis $(n=80149)$, surgeons with high volumes $(>15$ cholecystectomies/year) were associated with significantly decreased risk of a prolonged length of stay (odds ratio 0.91, $\mathrm{P}=0.022)$ and reduced risk of open conversion $(0.68$,

$\mathrm{P}<0.0001) .{ }^{10}$ In summary, differences in setting, participants, and the nationwide coverage of the current study might explain why our results indicated a consistent association between hospital volume and several outcome measures.

The underlying reason for the annual increase in the number of cholecystectomies is unclear, but is independent of total population growth (data not shown). An increase in gallstone disease through the last quarter of the 20th century has been described, ${ }^{21}$ yet a substantial change in prevalence during the study period seems unlikely. The introduction of laparoscopic cholecystectomy has increased the rates of cholecystectomy ${ }^{2021}$ and lowered clinical thresholds for cholecystectomy. ${ }^{22}$ In this study, the increase in cholecystectomy rate was confined to high volume centres without a reciprocal fall in numbers in centres with low or medium volumes. A previously private hospital was acquired by NHS Scotland and began providing an elective cholecystectomy service in 2002. This hospital was classified as a high volume centre, thus reducing the overall proportion of non-elective cholecystectomy in this group. Although this hospital contributed to the apparent increase in cholecystectomy rate in the high volume group, two of the other four high volume centres also showed considerable increases in numbers during the study period.

The total number of non-elective cholecystectomies increased over the study period, which probably reflected published evidence suggesting that early laparoscopic cholecystectomy in the management of cholecystitis is safe and shortens hospital stay. ${ }^{23}$ However, the total increase in cholecystectomy numbers cannot be explained solely by an increase in non-elective operating. It is more likely that the increase indicated a changing practice in high volume centres, which was only partly due to increased early cholecystectomy.

Variations in practice between healthcare providers are to be expected. These variations could reflect local healthcare needs, maximise local expertise, and do not necessarily result in differences in outcome. Nevertheless, although this study was not designed to prove causality, we did identify differences in practice between hospital volume groups that might relate to outcome. The proportion of procedures completed laparoscopically in high volume hospitals was significantly greater than those completed in low or medium volume centres. Although surgeons should be encouraged to convert to an open procedure if it is not safe to continue laparoscopically, the rate of primary open operations and open conversion have been suggested as indicators of quality. ${ }^{24}$ The overall ratio of laparoscopic to open procedures (3:1) across all hospitals in this study was comparable to that of other recently published series. ${ }^{25}$ Similarly, high volume hospitals made greater use of operative cholangiography and although its routine use is the subject of ongoing debate, it has been associated with a lower risk of common bile duct injury. ${ }^{27}$ Alternative explanations for hospital differences include variation in local case mix (we did not control for "difficult" cholecystectomies in the models), hospital facilities, and patient and surgeon attitudes. Operative cholangiography rates may indicate that hospital facilities and surgeon attitude are predominant factors. The influence of these different factors and their complex relations are the focus of ongoing work.

\section{Implications for clinicians and policymakers}

There are clear difficulties in reconciling outcome differences relating to institutional volume and the need to provide safe and cost effective healthcare close to patients' homes. The modelling in our study clearly shows that although significant risk differences between hospital volume groups exist, these differences are, for practical purposes, irrelevant for most patients in view of their low baseline risk. However, for high risk patients, and particularly those who are elderly or with comorbidity, hospital choice could be important even for elective procedures. Centres with low and medium volumes should be confident in their ability to deliver safe and effective care for the majority of patients, but might be advised to refer patients at high risk or with complex conditions to a high volume centre. Consideration should also be given to the current configuration of surgical services and whether elective and emergency surgical care of gallbladder disease is consistently patient focused.

\section{Unanswered questions and future research}

The factors that contribute to patient safety are numerous and hospital volume is only a surrogate for these fundamental indicators. Future research should focus on a more careful delineation of factors at patient, surgeon, and hospital levels, and on areas in which interventions in the process of care will yield greatest benefit. Volume-outcome research relies on high quality records rich in patient data that can be used to control for case mix, the gathering of which should become central to healthcare provision. Volume-outcome research should be presented in a comprehensible manner and be used as a starting point for important discussions regarding policy decisions, such as the centralisation and subspecialisation of services.

We thank Harry Purser (head of health intelligence, NHS Lothian) and Colin Houston (Information Services Division of NHS Scotland) for 


\section{What is already known on this topic}

Associations between hospital volume and outcome after specialist surgery are well defined and have guided healthcare service reconfiguration for the benefit of patients

The effect of hospital volume on outcome after low risk, high volume procedures (such as cholecystectomy) is unclear

Smaller studies have shown a weak link between increased hospital volume and reduced mortality after cholecystectomy

\section{What this study adds}

High volume centres have lower mortality, shorter lengths of stay, and reduced rates of reoperation and readmission after cholecystectomy. Although these relative risk differences are significant, they are irrelevant for most patients with a low baseline risk

For high risk patients, particularly those who are elderly or with comorbidity, hospital choice might be important even for elective procedures

Differences in hospital structures and processes should be examined; centralisation of care for higher risk patients could improve outcomes after cholecystectomy

providing access to the data and invaluable advice, and Olivia Swann for critically reviewing the manuscript.

Contributors: EMH is the guarantor and takes responsibility for the integrity and accuracy of these data and the final decision to submit for publication. EMH participated in the study design; data collection, processing, analysis, and interpretation; manuscript writing; and construction of tables and figures. SO'N participated in the systematic review, data interpretation, and manuscript writing. TM participated in the study design; data processing, analysis, and interpretation; and manuscript writing. PLW participated in data validation and manuscript writing. MD, SP-B, SJW, and OJG participated in the study design, data interpretation, and manuscript writing. The authors had full access to all data in the study.

Funding: The study was funded by the University of Edinburgh, which had no direct role in the study. The corresponding author had full independence from the funding source.

Competing interests: All authors have completed the Unified Competing Interest form at www.icmje.org/coi_disclosure.pdf (available on request from the corresponding author) and declare: support from the University of Edinburgh for the submitted work; no financial relationships with any organisations that might have an interest in the submitted work in the previous 3 years; MD, SP-B, SJW, and OJG work as consultant surgeons in one of the institutions included in this study, constituting a non-financial interest that may be relevant to the submitted work.

Ethical approval: Study approval was granted by the National Services Scotland Privacy Advisory Committee. No ethical approval was required. Patient consent: None required.

Data sharing: No additional data available.

1 Finks JF, Osborne NH, Birkmeyer JD. Trends in hospital volume and operative mortality for high-risk surgery. N Engl J Med 2011;364:2128-37.

2 Chowdhury MM, Dagash $\mathrm{H}$, Pierro A. A systematic review of the impact of volume of surgery and specialization on patient outcome. Br J Surg 2007;94:145-61.

3 Birkmeyer JD, Stukel TA, Siewers AE, Goodney PP, Wennberg DE, Lucas FL. Surgeon volume and operative mortality in the United States. N Engl J Med 2003;349:2117-27.

4 Gooiker GA, van Gijn W, Wouters MWJM, Post PN, van de Velde CJH, Tollenaar RAEM, et al. Systematic review and meta-analysis of the volume-outcome relationship in pancreatic surgery. Br J Surg 2011;98:485-94.

5 Mayer EK, Bottle A, Darzi AW, Athanasiou T, Vale JA. The volume-mortality relation for radical cystectomy in England: retrospective analysis of hospital episode statistics. BMJ 2010;340:c1128.

6 Ghaferi AA, Birkmeyer JD, Dimick JB. Complications, failure to rescue, and mortality with major inpatient surgery in Medicare patients. Ann Surg 2009;250:1029-34.

7 Schilling PL, Dimick JB, Birkmeyer JD. Prioritizing quality improvement in general surgery. J Am Coll Surg 2008;207:698-704.
8 Luft HS, Bunker JP, Enthoven AC. Should operations be regionalized? The empirical relation between surgical volume and mortality. N Engl J Med 1979;301:1364-9.

9 Murphy MM, Ng SC, Simons JP, Csikesz NG, Shah SA, Tseng JF. Predictors of major complications after laparoscopic cholecystectomy: surgeon, hospital, or patient? J Am Coll Surg 2010;211:73-80.

10 Csikesz NG, Singla A, Murphy MM, Tseng JF, Shah SA. Surgeon volume metrics in laparoscopic cholecystectomy. Dig Dis Sci 2009;55:2398-405.

11 Von Elm E, Altman DG, Egger M, Pocock SJ, Gøtzsche PC, Vandenbroucke JP. The Strengthening the Reporting of Observational Studies in Epidemiology (STROBE) statement: guidelines for reporting observational studies. Lancet 2007;370:1453-7.

12 Li B, Evans D, Faris P, Dean S, Quan H. Risk adjustment performance of Charlson and Elixhauser comorbidities in ICD-9 and ICD-10 administrative databases. BMC Health Serv Res 2008:8:12.

13 Kim SH, Park S-J, Lee S, Lee WJ, Park J-W, Hong EK, et al. Various liver resections using hanging maneuver by three glisson's pedicles and three hepatic veins. Ann Surg 2007;245:201-5.

14 Austin PC, Rothwell DM, Tu JV. A comparison of statistical modeling strategies for analyzing length of stay after CABG surgery. Health Serv Outcomes Res Methodol 2002;3:107-33.

15 King G, Tomz M, Wittenberg J. Making the most of statistical analyses: improving interpretation and presentation. Am J Pol Sci 2000;44:341-55.

16 Imai K, King G, Lau O. Zelig: everyone's statistical software. 2007. http://gking.harvard. edu/zelig.

17 Imai K, King G, Lau O. Toward a common framework for statistical analysis and development. J Computational Graphical Stat 2008;17:892-913.

18 Khuri SF, Daley J, Henderson W, Hur K, Hossain M, Soybel D, et al. Relation of surgical volume to outcome in eight common operations: results from the VA National Surgical Quality Improvement Program. Ann Surg 1999;230:414.

19 Buanes $\mathrm{T}$, Mjåland $\mathrm{O}$, Waage $\mathrm{A}$, Langeggen $\mathrm{H}$, Holmboe J. A population-based survey of biliary surgery in Norway. Surg Endosc 1998;12:852-5.

20 McMahon AJ, Fischbacher CM, Frame SH, MacLeod M. Impact of laparoscopic cholecystectomy: a population-based study. Lancet 2000;356:1632-7.

21 Bateson MC. Gallstones and cholecystectomy in modern Britain. Postgrad Med J 2000;76:700-3.

22 Escarce JJ, Chen W, Schwartz JS. Falling cholecystectomy thresholds since the introduction of laparoscopic cholecystectomy. JAMA 1995;273:1581.

23 Gurusamy K, Samraj K, Gluud C, Wilson E, Davidson BR. Meta-analysis of randomized controlled trials on the safety and effectiveness of early versus delayed laparoscopic cholecystectomy for acute cholecystitis. Br J Surg 2009;97:141-50.

24 Harboe KM, Bardram L. Nationwide quality improvement of cholecystectomy: results from a national database. Int J Qual Health Care 2011;23:565-73.

25 Rosenmüller M, Haapamäki M, Nordin P, Stenlund H, Nilsson E. Cholecystectomy in Sweden 2000-2003: a nationwide study on procedures, patient characteristics, and mortality. BMC Gastroenterol 2007;7:35.

26 David GG, Al-Sarira AA, Willmott S, Deakin M, Corless DJ, Slavin JP. Management of acute gallbladder disease in England. Br J Surg 2007;95:472-6.

27 Flum DR, Cheadle A, Prela C, Dellinger EP, Chan L. Bile duct injury during cholecystectomy and survival in medicare beneficiaries. JAMA 2003;290:2168.

Accepted: 08 April 2012

\section{Cite this as: BMJ 2012;344:e3330}

This is an open-access article distributed under the terms of the Creative Commons Attribution Non-commercial License, which permits use, distribution, and reproduction in any medium, provided the original work is properly cited, the use is non commercial and is otherwise in compliance with the license. See: http://creativecommons.org/licenses/by$\mathrm{nc} / 2.0 /$ and http://creativecommons.org/licenses/by-nc/2.0/legalcode. 


\section{Tables}

Table 1| Patient characteristics by hospital volume. Data are no (\%) unless otherwise stated

Hospital volume

Low ( $n=20$ 959) Medium ( $n=20$ 534) High ( $n=18$ 425)

\begin{tabular}{lccc} 
Age (years) & $54.0(30-77)$ & $52.0(28-74)$ & $54.0(29-77)$ \\
\hline Male:female ratio & $3.2: 1$ & $3.5: 1$ & $3.0: 1$ \\
\hline Admission type & & & \\
\hline Elective & $17374(82.9)$ & $16524(80.5)$ & $12381(67.2)$ \\
\hline Non-elective & $3585(17.1)$ & $4010(19.5)$ & $6044(32.8)$ \\
\hline Diagnosis & & & \\
\hline Cholelithiasis & $15924(76.0)$ & $14465(70.4)$ & $14694(79.8)$ \\
\hline Cholecystitis & $4095(19.5)$ & $4883(23.8)$ & $2003(10.9)$ \\
\hline Acute pancreatitis & $174(0.8)$ & $240(1.2)$ & $535(2.9)$ \\
\hline Other & $766(3.7)$ & $946(4.6)$ & $1193(6.5)$ \\
\hline
\end{tabular}

Deprivation (SIMD score)

\begin{tabular}{lccc}
\hline 1 (high) & $4006(19.1)$ & $6637(32.3)$ & $3379(18.3)$ \\
\hline 2 & $4995(23.8)$ & $4616(22.5)$ & $3894(21.1)$ \\
\hline 3 & $5336(25.5)$ & $3447(16.8)$ & $3468(18.8)$ \\
\hline 4 & $4234(20.2)$ & $2855(13.9)$ & $3637(19.7)$ \\
\hline 5 (low) & $2257(10.8)$ & $2921(14.2)$ & $3965(21.5)$ \\
\hline Missing data & $131(0.6)$ & $58(0.3)$ & $82(0.4)$ \\
\hline Morbidity & & & \\
\hline Charlson score $>0$ & $2505(12.0)$ & $2577(12.5)$ & $2339(12.7)$ \\
\hline Elixhauser score $>0$ & $2982(14.2)$ & $2973(14.5)$ & $2695(14.6)$ \\
\hline
\end{tabular}

*Data are median (interquartile range). 
Table 2| Operative procedures associated with cholecystectomy. Data are no (\%)

Hospital volume

Low ( $n=20$ 959) Medium ( $n=20$ 534) High $(n=18$ 425)

Approach

\begin{tabular}{lcccc}
\hline Open (including conversion) & $7409(35.3)$ & $5388(26.2)$ & $3521(19.1)$ & $<0.001$ \\
\hline Laparoscopic & $13550(64.7)$ & $15146(73.8)$ & $14904(80.9)$ & $<0.001$ \\
\hline Operative cholangiogram & $2345(11.2)$ & $1294(6.3)$ & $3911(21.2)$ & $<0.001$ \\
\hline
\end{tabular}

Endoscopic retrograde cholangiopancreatography

\begin{tabular}{lcccc}
\hline Preoperative & $2349(11.2)$ & $3271(15.9)$ & $2225(12.1)$ & $<0.001$ \\
\hline Postoperative & $979(4.7)$ & $1244(6.1)$ & $837(4.5)$ & $<0.001$ \\
\hline Preoperative and postoperative & $307(1.5)$ & $478(2.3)$ & $248(1.3)$ & $<0.001$ \\
\hline Bile duct exploration† & $591(2.8)$ & $675(3.3)$ & $652(3.5)$ & $<0.001$ \\
\hline
\end{tabular}

*Pearson's $\chi^{2}$ test.

†At the time of index cholecystectomy. 
Table 3| Unadjusted outcomes after cholecystectomy. Data are no (\%) unless stated otherwise

\begin{tabular}{|c|c|c|c|c|}
\hline & \multicolumn{3}{|c|}{ Hospital volume } & \multirow[t]{2}{*}{$\mathbf{P}$} \\
\hline & Low (n=20 959) & Medium ( $n=20534)$ & High $(n=18425)$ & \\
\hline Mortality* & $107(0.51)$ & $112(0.55)$ & $73(0.40)$ & $0.09 \dagger$ \\
\hline Reoperation at 30 days & 677 (3.23) & $954(4.65)$ & 607 (3.29) & $<0.001 \dagger$ \\
\hline Readmission at 30 days & $1583(7.55)$ & $1676(8.16)$ & $1403(7.61)$ & $0.024 \dagger$ \\
\hline $\begin{array}{l}\text { Length of stay (days; mean } \\
\text { (standard deviation)) }\end{array}$ & $2.99(3.59)$ & $3.09(3.74)$ & $2.59(3.41)$ & $<0.001 \ddagger$ \\
\hline
\end{tabular}

*Inpatient mortality and 30 day mortality combined.

†Pearson's $x^{2}$ test.

¥One way analysis of variance with Bonferroni correction of log transformed length of stay (separate comparisons: low $v$ medium volume, $\mathrm{P}=0.156$; low $v$ high volume, $\mathrm{P}<0.001$; medium $v$ high volume, $\mathrm{P}<0.001$ ). 


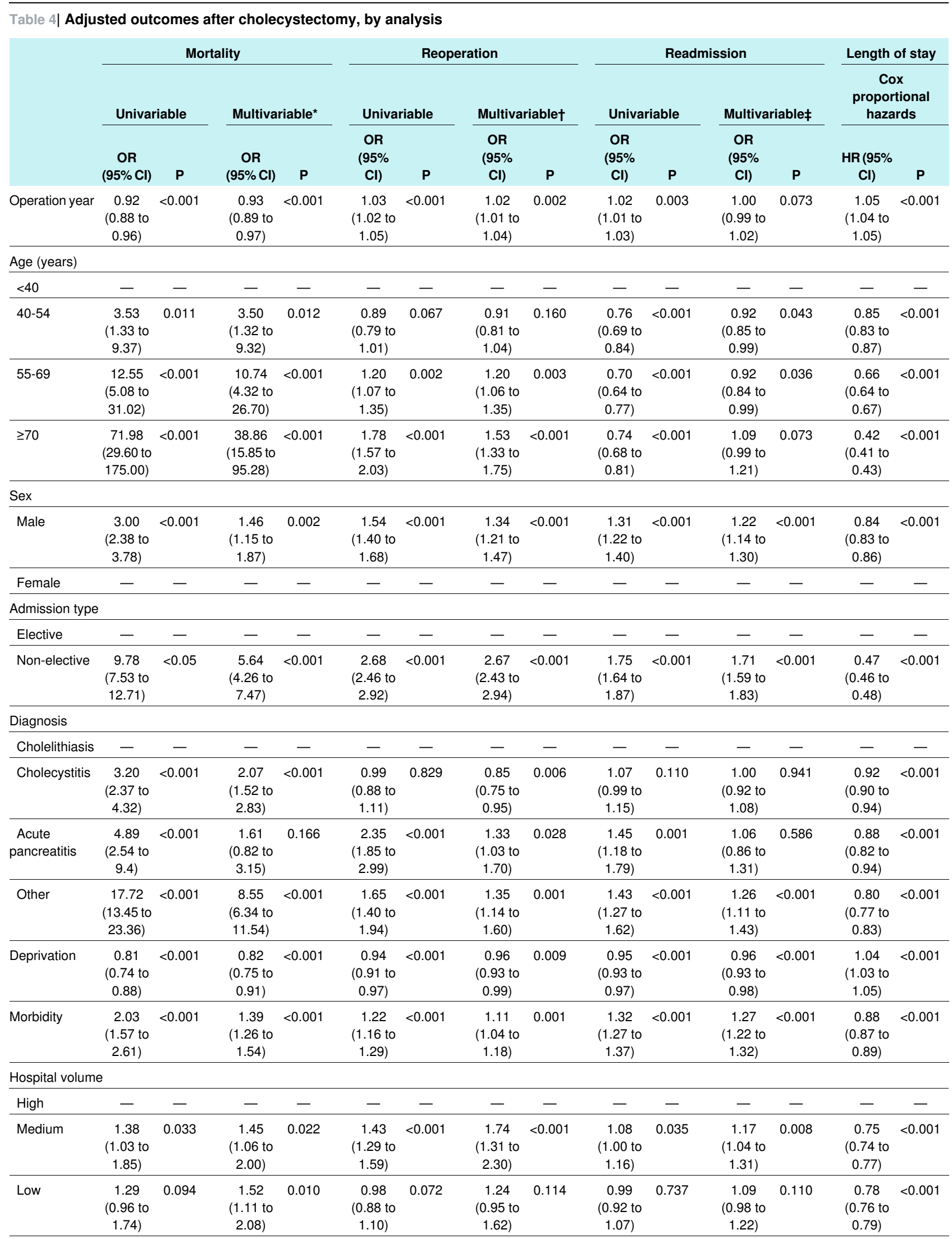

$\mathrm{OR}=\mathrm{odds}$ ratio. $\mathrm{HR}=$ hazard ratio for discharge.

${ }^{*}$ Fixed effects relogit model (Hosmer-Lemeshow test, $\chi^{2} 4.246, d f=8, P=0.834$; area under the receiver operator characteristic curve, $c$ statistic 0.92 ) 
Table 4 (continued)

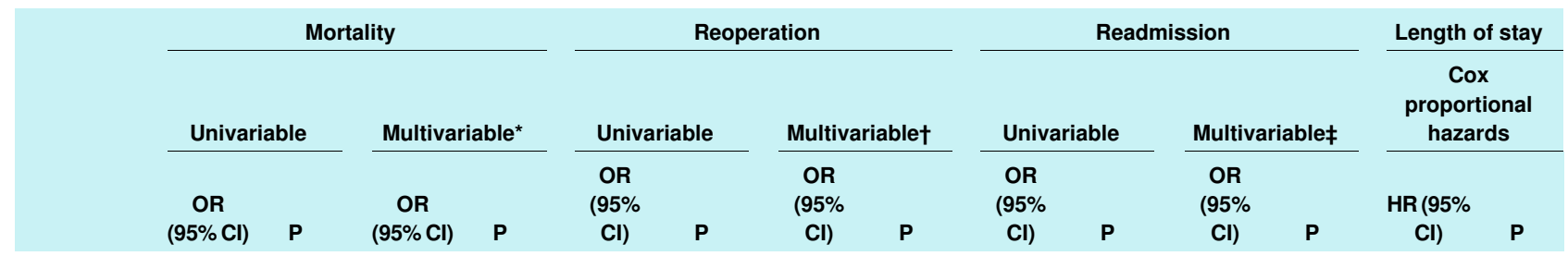

†Mixed effects hierarchical model (Hosmer-Lemeshow test, $\chi^{2} 11.276, \mathrm{df}=8, \mathrm{P}=0.187$ on fixed components; area under the receiver operator characteristic curve, c statistic 0.66).

$\ddagger$ Mixed effects hierarchical model (Hosmer-Lemeshow test, $\mathrm{X}^{2} 7.24, \mathrm{df}=8, \mathrm{P}=0.511$ on fixed components; area under the receiver operator characteristic curve, $\mathrm{C}$ statistic 0.60). 
Table 5| Risk modelling for probability of death after cholecystectomy using simulations. Operation year for all examples was 2007

\begin{tabular}{|c|c|c|c|c|c|c|c|}
\hline Risk factor & Example 1 & Example 2 & Example 3 & Example 4 & Example 5 & Example 6 & Example 7 \\
\hline Age (years) & $55-69$ & $55-69$ & $55-69$ & $55-69$ & $\geq 70$ & $\geq 70$ & $\geq 70$ \\
\hline Sex & Female & Female & Female & Female & Female & Female & Male \\
\hline $\begin{array}{l}\text { Non-elective } \\
\text { admission }\end{array}$ & No & Yes & No & Yes & No & Yes & Yes \\
\hline Diagnosis & Cholelithiasis & Cholecystitis & Cholelithiasis & Cholecystitis & Cholelithiasis & Cholecystitis & Cholecystitis \\
\hline $\begin{array}{l}\text { Deprivation (SIMD } \\
\text { score) }\end{array}$ & 3 & 3 & 1 & 1 & 1 & 1 & 1 \\
\hline $\begin{array}{l}\text { Morbidity (Charlson } \\
\text { score) }\end{array}$ & 0 & 0 & 4 & 4 & 4 & 4 & 4 \\
\hline \multicolumn{8}{|c|}{ Probability of death $(95 \% \mathrm{Cl})$} \\
\hline Low volume & $\begin{array}{c}0.00076(0.00049 \text { to } \\
0.00113)\end{array}$ & $\begin{array}{c}0.0088(0.0056 \text { to } \\
0.0133)\end{array}$ & $\begin{array}{c}0.0043(0.0024 \text { to } \\
0.0072)\end{array}$ & $\begin{array}{c}0.048(0.027 \text { to } \\
0.078)\end{array}$ & $\begin{array}{c}0.0153(0.0086 \text { to } \\
0.0251)\end{array}$ & $\begin{array}{c}0.151(0.094 \text { to } \\
0.226)\end{array}$ & $\begin{array}{c}0.206(0.132 \text { to } \\
0.298)\end{array}$ \\
\hline Medium volume & $\begin{array}{c}0.00073(0.00047 \text { to } \\
0.00110)\end{array}$ & $\begin{array}{c}0.0085(0.0053 \text { to } \\
0.0128)\end{array}$ & $\begin{array}{c}0.0041(0.0023 \text { to } \\
0.0068)\end{array}$ & $\begin{array}{c}0.046(0.026 \text { to } \\
0.073)\end{array}$ & $\begin{array}{c}0.0146(0.0083 \text { to } \\
0.0238)\end{array}$ & $\begin{array}{c}0.146(0.091 \text { to } \\
0.216)\end{array}$ & $\begin{array}{c}0.199(0.129 \text { to } \\
0.285)\end{array}$ \\
\hline High volume & $\begin{array}{c}0.00051(0.00032 \text { to } \\
0.00076)\end{array}$ & $\begin{array}{c}0.0059(0.0036 \text { to } \\
0.0090)\end{array}$ & $\begin{array}{c}0.0029(0.0015 \text { to } \\
0.0048)\end{array}$ & $\begin{array}{c}0.032(0.018 \text { to } \\
0.054)\end{array}$ & $\begin{array}{c}0.0102(0.0056 \text { to } \\
0.0170)\end{array}$ & $\begin{array}{c}0.106(0.063 \text { to } \\
0.165)\end{array}$ & $\begin{array}{c}0.147(0.090 \text { to } \\
0.222)\end{array}$ \\
\hline \multicolumn{8}{|c|}{ Absolute risk difference $(95 \% \mathrm{Cl})$} \\
\hline Low $v$ high volume* & $\begin{array}{c}0.00026(0.00006 \text { to } \\
0.00051)\end{array}$ & $\begin{array}{c}0.0030(0.0007 \text { to } \\
0.0059)\end{array}$ & $\begin{array}{c}0.0014(0.0003 \text { to } \\
0.0031)\end{array}$ & $\begin{array}{c}0.016(0.003 \text { to } \\
0.032)\end{array}$ & $\begin{array}{c}0.0051(0.0011 \text { to } \\
0.0107)\end{array}$ & $\begin{array}{c}0.045(0.010 \text { to } \\
0.087)\end{array}$ & $\begin{array}{c}0.059(0.014 \text { to } \\
0.110)\end{array}$ \\
\hline $\begin{array}{l}\text { Medium } v \text { high } \\
\text { volume† }\end{array}$ & $\begin{array}{c}0.00023(0.00003 \text { to } \\
0.00047)\end{array}$ & $\begin{array}{c}0.0026(0.0003 \text { to } \\
0.0054)\end{array}$ & $\begin{array}{c}0.0013(0.0002 \text { to } \\
0.0027)\end{array}$ & $\begin{array}{c}0.013(0.002 \text { to } \\
0.028)\end{array}$ & $\begin{array}{c}0.0044(0.0006 \text { to } \\
0.0096)\end{array}$ & $\begin{array}{c}0.040(0.005 \text { to } \\
0.079)\end{array}$ & $\begin{array}{c}0.051(0.007 \text { to } \\
0.024)\end{array}$ \\
\hline \multicolumn{8}{|c|}{ Number needed to treat to harm $(95 \% \mathrm{Cl})$} \\
\hline Low $v$ high volume* & $\begin{array}{c}3871 \text { (1963 to } 17 \\
118)\end{array}$ & 338 (171 to 1491$)$ & 691 (325 to 3242) & 64 (31 to 294) & 196 (94 to 908 ) & 22 (11 to 97 ) & 17 (9 to 74$)$ \\
\hline $\begin{array}{l}\text { Medium } v \text { high } \\
\text { volume }\end{array}$ & $\begin{array}{c}4431 \text { (2120 to } 34 \\
681)\end{array}$ & 387 (186 to 781$)$ & 798 (366 to 6216 ) & 74 (35 to 557 ) & 226 (104 to 1726$)$ & 25 (13 to 185$)$ & 19 (10 to 143$)$ \\
\hline
\end{tabular}

${ }^{*} \mathrm{P}=0.010$.

$\dagger P=0.023$ 


\section{Figures}
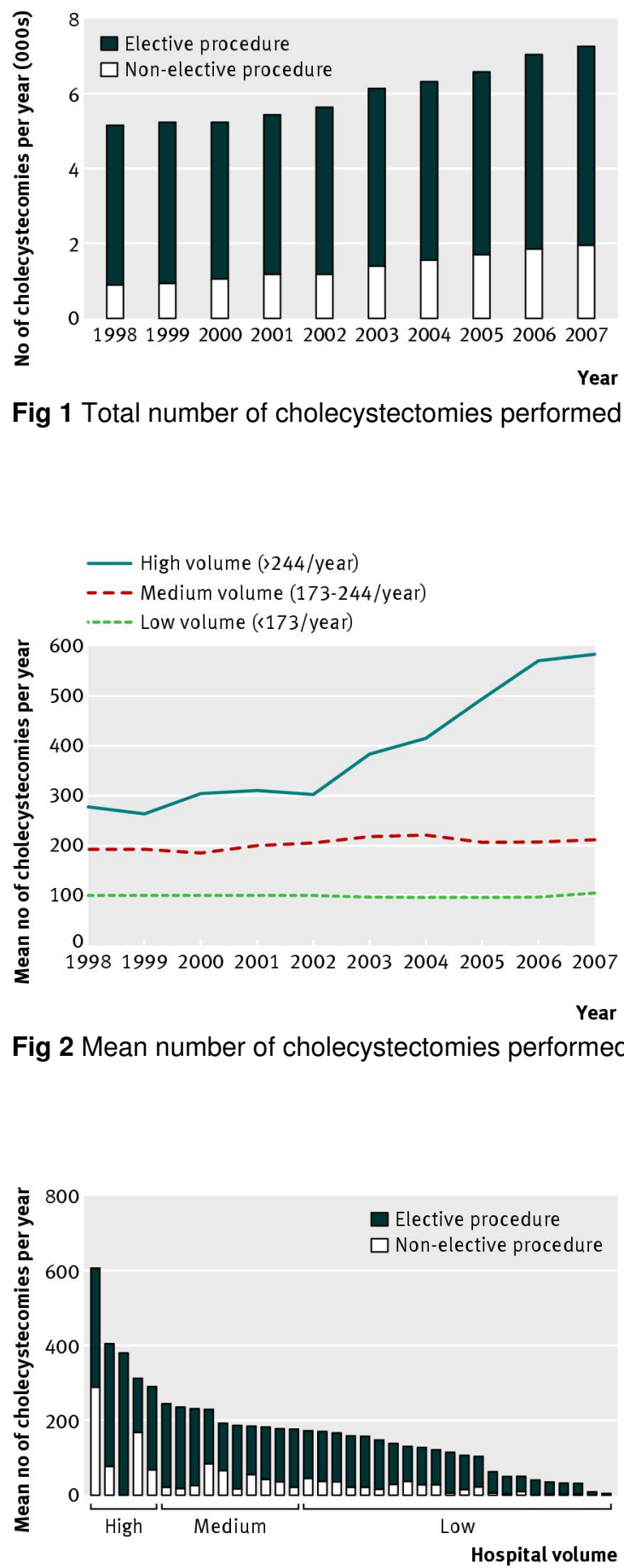

Fig 3 Mean annual hospital volume per institution. Each bar represents one of 37 hospitals in Scotland that performed cholecystectomies during the study period 

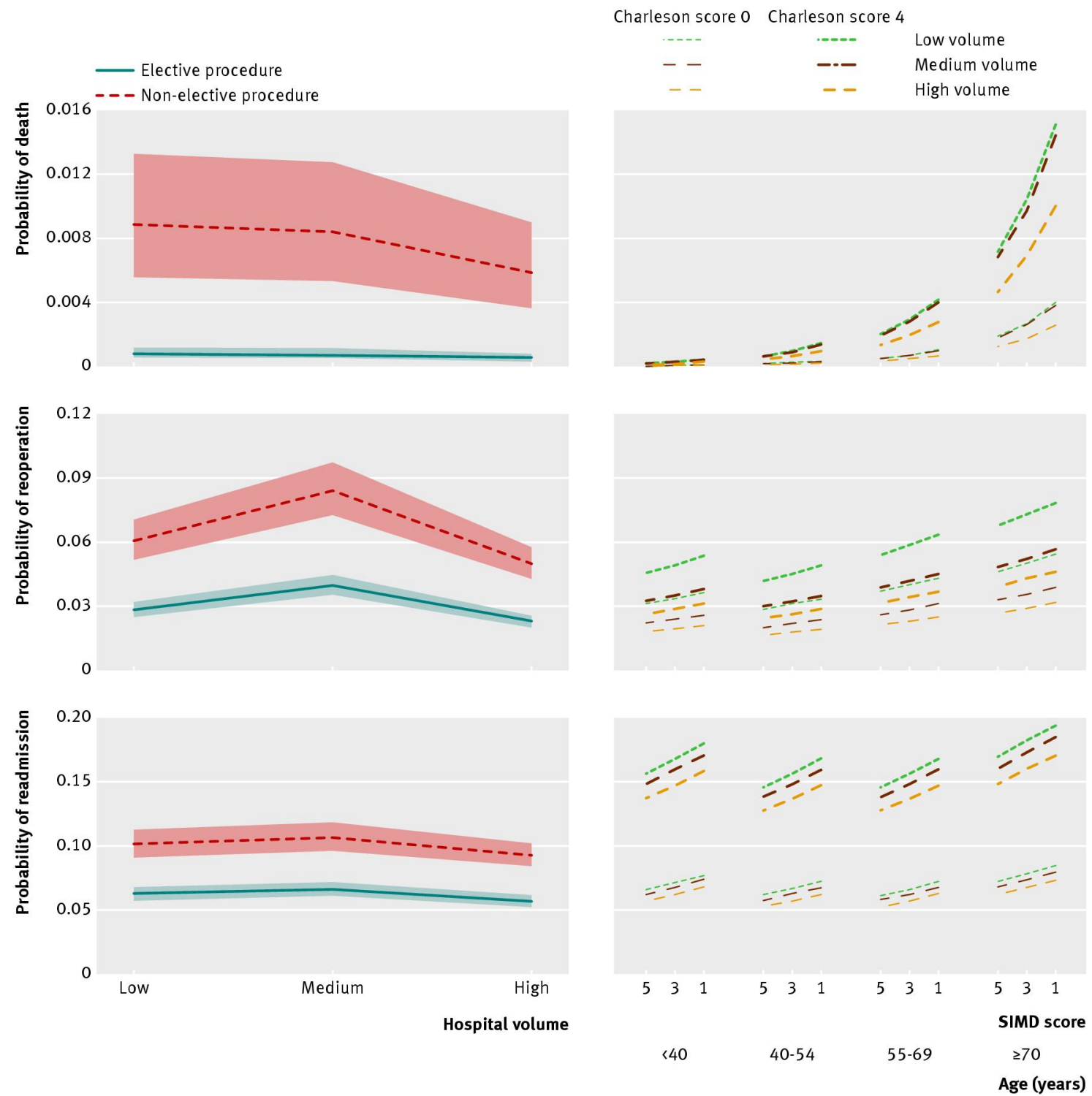

Fig 4 Risk modelling using simulation procedures. Left panel shows probability of death, reoperation, and readmission by hospital volume group for elective (example 1 in table 5) and non-elective (example 2 in table 5) cholecystectomy in patients at standard risk (operation year 2007, age 55-69 years, female, SIMD score 3, Charlson score 0). Shaded area=95\% confidence interval. Right panel shows probability of death, reoperation, and readmission in the elective setting by hospital volume group as a function of age, SIMD score, and Charlson score (operation year 2007, female, elective admission, cholelithiasis diagnosis). 